Paper

\title{
A method for estimating of synaptic connectivity from spike data of multiple
}

\section{neurons}

\author{
Ryota Kobayashi ${ }^{1,2}$ and Katsunori Kitano ${ }^{3 a}$ ) \\ ${ }^{1}$ Principles of Informatics Research Division, National Institute of Informatics \\ 2-1-2 Hitotsubashi, Chiyoda-ku, Tokyo, Japan \\ ${ }^{2}$ Department of Informatics, SOKENDAI (The Graduate University for \\ Advanced Studies) \\ 2-1-2 Hitotsubashi, Chiyoda-ku, Tokyo, Japan \\ ${ }^{3}$ Department of Human and Computer Intelligence, Ritsumeikan University \\ 1-1-1 Nojihigashi, Kusatsu, Shiga 525-8577, Japan \\ a)kitano@ci.ritsumei.ac.jp
}

Received August 20, 2015; Revised November 13, 2015; Published April 1, 2016

\begin{abstract}
Investigation of synaptic connectivity between neurons is essential for understanding information processing mechanisms in the brain because this connectivity determines how spikes propagate in a neural circuit. Recent advances in experimental technology have enabled simultaneous recording of the spiking activity from hundreds of neurons. In this study, we propose a method for estimating synaptic connectivity using the spike data from multiple neurons. We first demonstrated that this method can perfectly estimate synaptic connectivity using synthetic spike data in an ideal condition. Subsequently, this method is applied to more realistic spike data generated by a large-scale network of Hodgkin-Huxley neurons. The results suggest that our estimation method is superior to a conventional method in identifying synaptic connectivity when the spiking activity from a large number of neurons is available for the analysis.
\end{abstract}

Key Words: neuroscience, synaptic connectivity, connectivity inference, maximum likelihood estimation, Poisson process, large-scale network simulation

\section{Introduction}

The brain consists of numerous nerve cells (neurons) that transmit information through the propagation of action potentials (spikes). To understand information processing mechanisms in the brain, it is essential to investigate synaptic connectivity because it determines how the spikes propagate through the neurons. Recent advances in experimental technology, such as $\mathrm{Ca}^{2+}$ imaging techniques [1] and multi-electrode arrays $[2,3]$, enable us simultaneously to record the spiking activity of hundreds of neurons. However, a method for inferring synaptic connectivity from the spike data of multiple neurons 
has not been established.

Cross-correlation method [4] is the most common method to infer the connectivity between neurons from experimental data $[2,4]$, which is based on the correlation between two spike trains. Subsequently, transfer entropy [5] that quantifies information flow from a time series to the other one was proposed. The transfer entropy has been also applied for estimating the synaptic connectivity [3,6]. Recently, we developed a method for estimating synaptic connectivity based on the coupled escape rate model (CERM), and the estimation performance was evaluated by analyzing a simulated data generated from a large-scale network of detailed neuron models [7]. The CERM performed better than the cross-correlation and the transfer entropy method for the simulated data. All the previous methods are based on the pairwise analysis, which means that the synaptic connectivity between two neurons is estimated from spike trains of the corresponding two neurons. The effect of the other neurons on the two neurons is neglected in these method.

In this study, we extend the CERM to simultaneously estimate the synaptic connectivity from spike data of more than two neurons. First, we tested the extended method using synthetic spike data generated by a small network of the CERM neurons. After this verification, the method was applied to more realistic spike data generated by the large-scale network model, and the estimation performance of the extended method was compared to that of the original CERM.

\section{Estimating synaptic connectivity from neuronal activity}

We aimed to estimate synaptic connectivity among neurons from multineuronal spike data (Fig. 1). To accomplish this goal, we developed a probabilistic model of neuronal activity and determine the model parameters from the spike data. Synaptic connectivity is inferred from the model parameters.

Spike Data

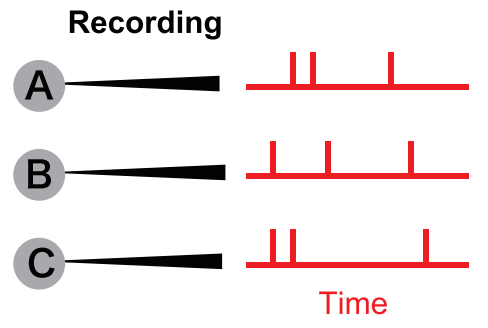

\section{Synaptic Connection}

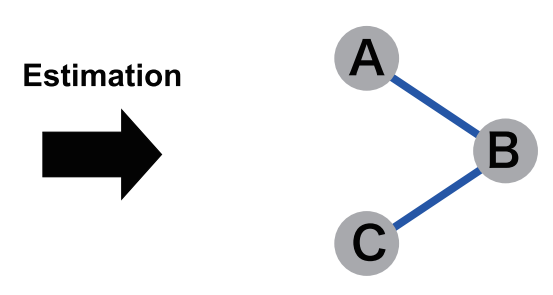

Fig. 1. Estimating synaptic connectivity from multineuronal spike data assuming that we recorded the spike times from $N$ neurons $(N=3$ in this figure). The issue considered here was to estimate from the spike data (red) whether or not there were synaptic connections (blue) between the neurons.

\subsection{Coupled escape rate model}

Coupled escape rate model (CERM) [7] was used to describe the spiking activity of recorded neurons. The spiking activity was described by the Poisson process, which means that the spike probability of the $i$-th neuron in an interval $[t, t+\Delta t]$ is

$$
\begin{aligned}
& P(\text { Neuron } i \text { generates a spike in }[t, t+\Delta t])=\lambda_{i}(t) \Delta t, \\
& \lambda_{i}(t)=\exp \left[u_{i}+\alpha_{i} x_{i}(t)+\sum_{j \neq i} J_{i j} s_{i j}(t)\right],
\end{aligned}
$$

where $\lambda_{i}(t)$ and $x_{i}(t)$ are the firing rate and waveform of spike-after potential of the $i$-th neuron, respectively; and $s_{i j}(t)$ is the waveform of postsynaptic potential from neurons $j$ to $i$. The term $u_{i}+\alpha_{i} x_{i}(t)+\sum_{j \neq i} J_{i j} s_{i j}(t)$ represents a rescaled membrane potential of the $i$-th neuron measured from the spike threshold. The exponential function was used to link the spike probability to the membrane potential. It has been shown that the exponential link function is a good model for neurons recorded in a slice experiment (in vitro) $[8,9]$ and for a biophysical Hodgkin-Huxley type model [10]. 
The spike-after potential $x_{i}(t)$ and postsynaptic potential $s_{i j}(t)$ are described by the following differential equations:

$$
\begin{aligned}
& \frac{d x_{i}(t)}{d t}=-\frac{x_{i}}{\tau_{m}}+\sum_{t_{i, k}<t} \delta\left(t-t_{i, k}\right), \\
& \frac{d s_{i j}(t)}{d t}=-\frac{s_{i j}}{\tau_{s}}+\sum_{t_{j, k}<t} \delta\left(t-t_{j, k}\right),
\end{aligned}
$$

where $\tau_{m}$ and $\tau_{s}$ are the membrane time constant and synaptic time constant, respectively, $t_{i, k}$ is the $k$-th spike time of the $i$-th neuron, and $\delta(t)$ is the Dirac delta function.

\subsection{Parameter estimation}

The CERM parameters $\left(u_{i}, \alpha_{i}, J_{i j}\right)(i, j=1, \cdots, N)$ were estimated by the maximum likelihood method. The likelihood function $L$ of the recorded $N$ neurons, i.e., the probability for obtaining the observed spike data, can be written as the product,

$$
\begin{aligned}
& L=\prod_{i=1}^{N} L_{i}\left(\vec{\theta}_{i}\right), \\
& L_{i}=\exp \left(-\int_{0}^{T} \lambda_{i}(s) d s\right) \prod_{k=1}^{M_{i}} \lambda_{i}\left(t_{i, k}^{-}\right),
\end{aligned}
$$

where $L_{i}$ is the likelihood of the $i$-th neuron [11], $\vec{\theta}_{i}:=\left(u_{i}, \alpha_{i}, J_{i 1}, J_{i 2}, \cdots, J_{i N}\right)$ are the CERM parameters for the $i$-th neuron, $t_{i, k}^{-}:=\lim _{\epsilon \rightarrow-0} t_{i, k}$ is the left-sided limit of $t_{i, k}, M_{i}$ is the number of spikes from the $i$-th neuron, and $T$ is the recording time. The log likelihood function $l$ is written as

$$
\begin{aligned}
& l=\log L=\sum_{i=1}^{N} l_{i}\left(\vec{\theta}_{i}\right), \\
& l_{i}\left(\vec{\theta}_{i}\right)=\log L_{i}=\sum_{k=1}^{M_{i}} \log \left(\lambda_{i}\left(t_{i, k}^{-}\right)\right)-\int_{0}^{T} \lambda_{i}(s) d s,
\end{aligned}
$$

where $l_{i}\left(\vec{\theta}_{i}\right)$ is the $\log$ likelihood function of the $i$-th neuron. Because of the separation in the variables in Eq. (7), the CERM parameters $\vec{\theta}_{i}$ were determined by maximizing the individual log likelihood function $l_{i}$ instead of maximizing the full log likelihood function $l$.

The log likelihood function was maximized by using Levenberg-Marquardt algorithm [12]:

$$
\vec{\theta}_{i}^{(k+1)}=\left\{H\left(l_{i}\right)+c_{\mathrm{LM}} \operatorname{diag}\left(H\left(l_{i}\right)\right)\right\}^{-1} \nabla l_{i}\left(\vec{\theta}_{i}^{(k)}\right),
$$

where $\vec{\theta}_{i}^{(k)}$ is the $k$-th iterated values of $\vec{\theta}_{i}, c_{\mathrm{LM}}$ is the damping parameter, and $\nabla l_{i}, H\left(l_{i}\right)$ are the gradient and the Hessian of the log-likelihood with respect to $\vec{\theta}_{i}$, respectively. The matrix $\operatorname{diag}\left(H\left(l_{i}\right)\right)$ is the diagonal part of the Hessian matrix; i.e., $\operatorname{diag}\left(H\left(l_{i}\right)\right)_{i j}=H\left(l_{i}\right)_{i i}$ for the diagonal part $i=j$, otherwise $\operatorname{diag}\left(H\left(l_{i}\right)\right)_{i j}=0$, where $A_{i j}$ represents the $i, j$ element of the matrix $A$. The iteration was terminated if the parameter change in a iteration $\left|\vec{\theta}_{i}^{(k+1)}-\vec{\theta}_{i}^{(k)}\right|$ was smaller than $10^{-3}$.

\subsection{Estimation of synaptic connectivity}

We aimed to estimate synaptic connectivity from multineuronal spike data. The synaptic connectivity was reconstructed by thresholding the connectivity parameters $J_{i j}$, i.e., we inferred that a synapse was connected from neurons $j$ to $i$, if $J_{i j}$ was larger than the threshold $J_{\mathrm{th}}$. The reconstructed connectivity can be asymmetric, i.e., the estimated synaptic connection between neuron $i$ and $j$ can be two-way, one-way, or no connection.

The quality of the synaptic reconstruction was evaluated using the Matthews Correlation Coefficient (MCC) $[7,13]$, which is an extension of the correlation coefficient to binary variables. MCC between two binary vectors $(\vec{v}, \vec{w})$ is defined as follows: 


$$
M C C=\frac{T P \times T N-F P \times F N}{\sqrt{(T P+F P)(T P+F N)(T N+F P)(T N+F N)}},
$$

where, $T P, T N, F P$, and $F N$ are the number of true positive, true negative, false positive, and false negative values, respectively. $\mathrm{MCC}$ is similar to the correlation coefficient in the sense that it ranges from -1 to 1 , with $M C C=1$ means the perfect match, $\vec{v}=\vec{w}, M C C=0$ meaning that the match is not better than the random prediction, and $M C C=-1$ meaning that the binary vector is the reverse of the other one, $\vec{v}=-\vec{w}$.

\section{Simulation model of a local neural circuit}

We simulated the spiking activity in a local neural circuit $[7,14]$ to evaluate the estimation performance for synaptic connectivity. The simulated model and its parameters are the same as those used in [7]. Thus, we provide only a brief overview of the simulated model without going into details.

The local circuit model consisted of 2 neural populations; excitatory and inhibitory populations (Fig. 2). There were a total of 5,120 Hodgkin-Huxley type model neurons, with 4,096 excitatory and 1,024 inhibitory neurons. Each neuron received not only the synaptic input from other neurons in the circuit (synaptic input $I_{\text {syn }}$ ), but also input from the neurons in other cortical areas (background input $\left.I_{\mathrm{bg}}\right)$.

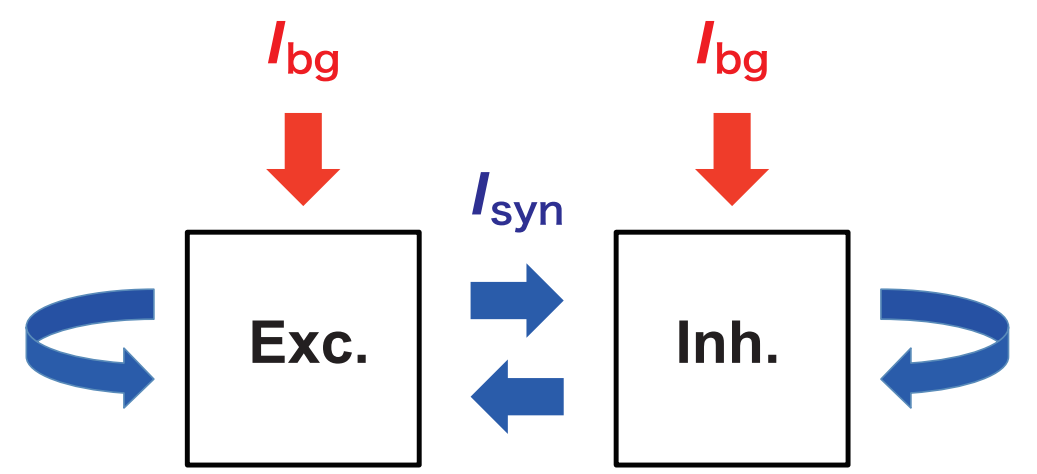

Fig. 2. Local neural circuit model. The local circuit was composed of excitatory (Exc.) and inhibitory (Inh.) populations. Each neuron received the synaptic input $\left(I_{\mathrm{syn}}\right.$, blue) from the local circuit and background input $\left(I_{\mathrm{bg}}\right.$, red) from the other areas.

Excitatory neurons were modeled as regular-spiking neurons [15]:

$$
C_{\mathrm{m}} \frac{d V}{d t}=-I_{\mathrm{L}}-I_{\mathrm{Na}}-I_{\mathrm{K}}-I_{\mathrm{M}}+I_{\mathrm{syn}}+I_{\mathrm{bg}},
$$

where $V$ is the membrane voltage of an excitatory neuron, and $I_{\mathrm{L}}, I_{\mathrm{Na}}, I_{\mathrm{K}}$, and $I_{\mathrm{M}}$ are the leak, spikegenerating sodium, delayed rectifier potassium, and muscarinic potassium ionic current, respectively. Inhibitory neurons were modeled as fast-spiking neurons [16]:

$$
C_{\mathrm{m}} \frac{d V}{d t}=-I_{\mathrm{L}}-I_{\mathrm{Na}}-I_{\mathrm{Kv}}+I_{\mathrm{syn}}+I_{\mathrm{bg}}
$$

where $V$ is the membrane voltage of an inhibitory neuron and $I_{\mathrm{Kv}}$ is an ionic current through Kv3.1-Kv3.2 potassium channels. The synaptic current $I_{\mathrm{syn}}$ is the sum of the excitatory and inhibitory currents from the presynaptic neurons. Excitatory synaptic currents consist of AMPA and NMDA currents, whereas inhibitory synaptic currents consist of GABA current. For simplicity, we set the model such that excitatory postsynaptic conductance (EPSC) and inhibitory postsynaptic conductance (IPSC) had uniform values for connected synapses, i.e., $g_{\mathrm{AMPA}}=0.028 \mathrm{mS} / \mathrm{cm}^{2}$ and $g_{\mathrm{NMDA}}=0.00085 \mathrm{mS} / \mathrm{cm}^{2}$ for excitatory synapses and $g_{\mathrm{GABA}}=0.017 \mathrm{mS} / \mathrm{cm}^{2}$ for inhibitory synapses. The excitatory postsynaptic potential (EPSP) was $1.0 \mathrm{mV}$ when the membrane voltage is fixed at the resting voltage. If there is no connection between neurons, the synaptic conductance was set to $0 \mathrm{mS} / \mathrm{cm}^{2}$. We simulated the local circuit with "small-world" synaptic connectivity whose 
network structure exhibits high clustering coefficient and small shortest path length. This circuit was generated by the following two steps. First, the excitatory and inhibitory neurons were arranged in a two-dimensional lattice, and all the neighboring neurons within a distance of 4 lattice-lengths were synaptically connoted. Second, the excitatory connections were rewired randomly with the probability $p_{r}$. In the present study, we focused on networks with the rewiring probabilities of $p_{r}=0.1,0.25$, and 0.5 .

It should be noted that recent experiments revealed that the amplitudes of EPSPs between cortical neurons obeys skewed distributions, such as log-normal distribution [17]. However, the distribution of EPSP amplitudes can be approximated by few strong EPSPs and a number of weak EPSPs [18]. Therefore, the simulated network is reasonable for validating the methods for detecting the strong synapses from spike data.

\section{Estimating synaptic connectivity from simulated neuronal activity}

First, we demonstrated that the proposed method (Section 2) could estimate the synaptic connectivity from the spike data generated by a small network of CERM neurons. The method was subsequently applied to the spike data generated by a local neural circuit model (Section 3), and we examined the estimation performance under more realistic conditions.

\subsection{Small network of CERM neurons}

We simulated a small neuronal network to test the validity of the method for estimating synaptic connectivity. The simulated network consisted of $N=10$ excitatory neurons, whose spiking activity is described by CERM $(1,2)$. The parameters are set to a fixed value for all the neurons, i.e., $u_{i}=-5.3, \alpha_{i}=-5.0$, and $\tau_{m}=10 \mathrm{~ms}$. The neurons were randomly connected with the coupling probability being 0.1 . For simplicity, the connectivity and synaptic time constant were uniform values, such that $J_{i j}=1.0, \tau_{s}=10 \mathrm{~ms}$, if there was a synaptic connection from neurons $j$ to $i$. Spike data was generated by simulating the CERM network with the time step of $\delta t=1 \mathrm{~ms}$.

Here, we consider the situation in which spike times of all the neurons are recorded. The CERM parameters $\left\{u_{i}, \alpha_{i}, J_{i j}\right\}$ (110 parameters) were estimated from spike data using the maximum likelihood method (Section 2.2). The true connectivity matrix $J_{i j}$ used in the simulation was compared with its estimates (Fig. 3A). The estimate obtained from 300-seconds-long spike data was better than that obtained from 30-seconds-long spike data. The mean squared errors were 0.62 and 0.21 for the $30 \mathrm{sec}$ data and for the $300 \mathrm{sec}$ data, respectively. Further, the distribution of the elements of the estimated connectivity matrix for the connected neuron pairs was compared with the distribution for the unconnected neuron pairs (Fig. 3B). The mean and standard deviation of the estimated connectivity were $0.75 \pm 0.52$ and $1.01 \pm 0.14$ for the connected pairs obtained from the $30 \mathrm{sec}$ and the $300 \mathrm{sec}$ data $(n=14)$, and $-0.07 \pm 0.63$ and $0.00 \pm 0.22$ for the unconnected pairs $(n=76)$. The estimate from the $30 \mathrm{sec}$ data showed a significant overlap between the distributions for the connected pairs and for the unconnected pairs, whereas the estimate from the $300 \mathrm{sec}$ data showed no overlap (Fig. 3B). This finding means that synaptic connectivity can be perfectly estimated if the recording time $T$ is 300 sec and the optimal threshold $J_{t h}$ is tuned. To quantify the overlap, we calculated MCC between the elements of the true connectivity matrix and their optimal estimates. A high MCC value implies that there is a small overlap and MCC value of 1 indicates no overlap. The MCC was 0.72 for the 30 sec case, 1 for the $300 \mathrm{sec}$ case. The result suggests that the long spike data provides accurate estimation results, and 300 seconds is sufficient time to reconstruct the synaptic connectivity from spike data in this simulated example. We also analyzed spike data generated by a small CERM network with log-normal connectivity. Preliminary result suggests that the proposed method can detect strong synaptic connections (Data not shown).

The simulated spike data used here were ideal for estimating synaptic connectivity. First, it was assumed that the spiking activity of all neurons was observable, whereas only a fraction of neurons in the brain are experimentally recordable. Second, the simulated network consisted of only the excitatory neurons that increase the probability of postsynaptic neuron spike generation. However, the 
brain consists of not only excitatory neurons, but also inhibitory neurons that decrease the probability of post-synaptic neuron spike generation. Third, the exponential postsynaptic potential was used to describe the synaptic input in the network model. The synaptic input may not be captured by a simple exponential function because it is mediated by various types of synapses (AMPA, NMDA, and GABA [11]). Estimation performances in more realistic situations are investigated in Section 4.2.
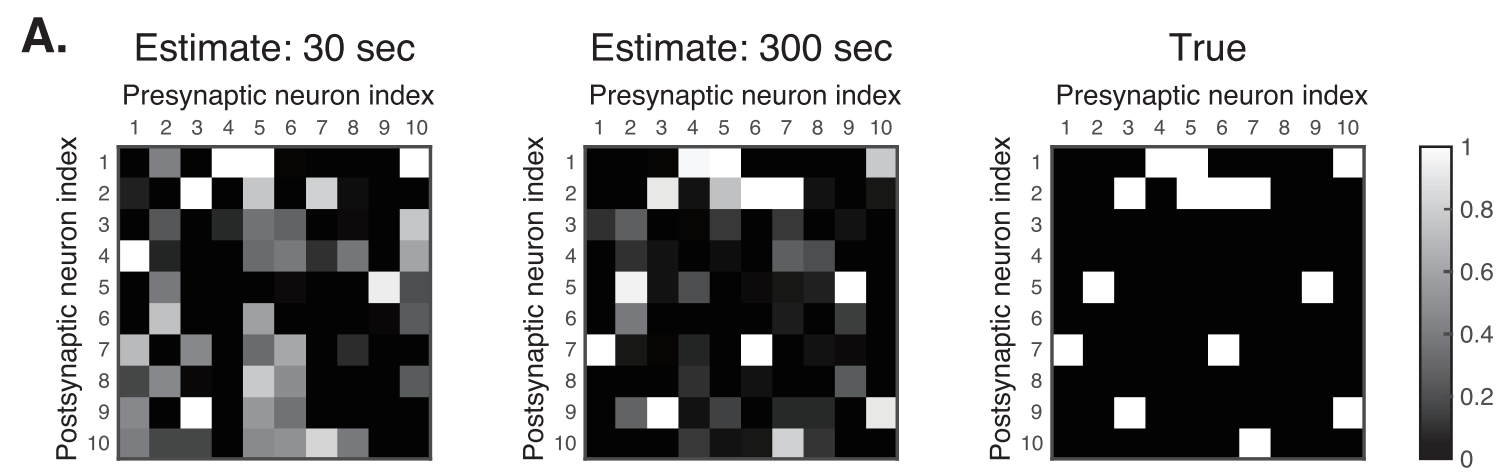

\section{B. Estimate: $30 \mathrm{sec}$}
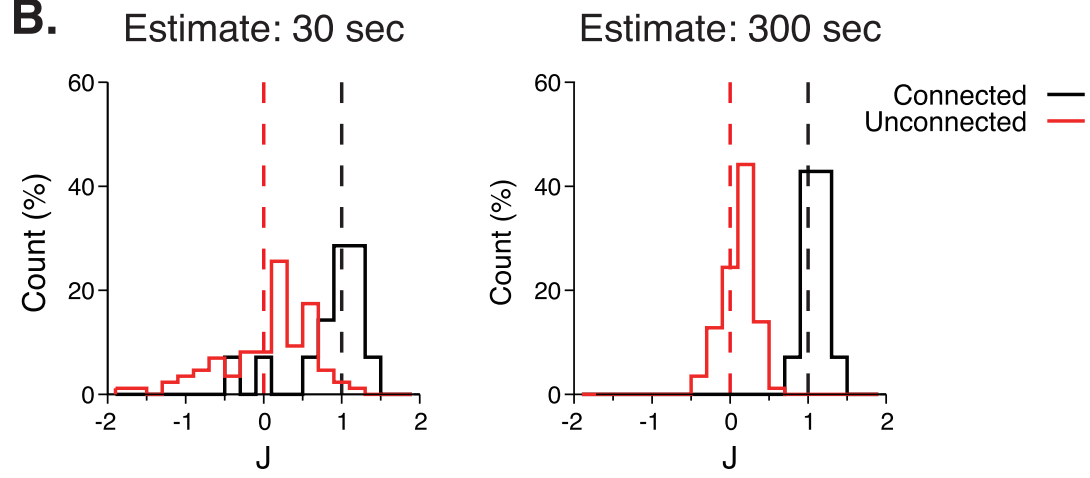

Fig. 3. Estimation of synaptic connectivity from spike data generated by a small random network of CERM neurons. (A) Estimation results. The true connectivity matrix $J_{i j}$ (right) was compared with its estimate from the spike data of $30 \mathrm{sec}$ (left) and that of $300 \mathrm{sec}$ (center). (B) Distribution of the elements of the connectivity matrix. Black represents the distribution for synaptically connected neuron pairs, whereas red represents the distribution for the unconnected pairs. Dashed lines represent the true connectivity for the connected synapses (black: $J_{i j}=1.0$ ) and for unconnected synapses (red: $\left.J_{i j}=0.0\right)$.

\subsection{Large-scale network of Hodgkin-Huxley type neurons}

We simulated a large-scale network of Hodgkin-Huxley type neurons to generate realistic spike data from the brain. The network consisted of 4,096 excitatory model neurons and 1,024 inhibitory model neurons that were arranged in two-dimensional lattice of $64 \times 64$ and $32 \times 32$, respectively. The network topology of the synaptic connectivity was small-world. First, the adjacent neuron pairs within 4 units of the lattice were connected synaptically. Then, the excitatory connections were rewired with the probability $p_{r}=0.1,0.25$ and 0.5 . See Section 3 and our previous report [7] for a detailed description of the simulated network.

We considered a partial observation scenario, i.e., the spiking activity of 49 excitatory neurons ( $0.96 \%$ of the total population) was recorded for 300 seconds. The recorded neurons were located in the center of the population at the coordinates $\left(32+2 k_{x}, 32+2 k_{y}\right)\left(k_{x}, k_{y} \in\{-3,-2,-1,0,1,2,3\}\right)$.

The synaptic connectivity was estimated by the original CERM and the extended CERM. In the original CERM, each neuron pair (neuron $i$ and $j$ ) from the 49 neurons was picked up and the corresponding connectivity $J_{i j}$ was estimated from spike data of the two neurons. On the other hand, the connectivity matrix was simultaneously estimated from spike data of all the recorded neurons in the extended CERM. The distribution of the estimated connectivity $J_{i j}$ for synaptically 
A.

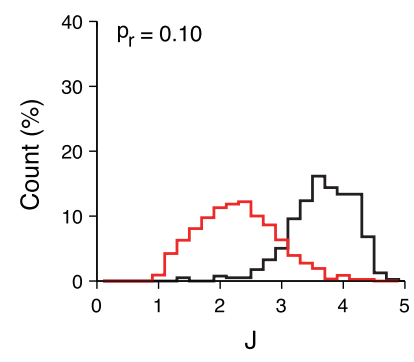

B.

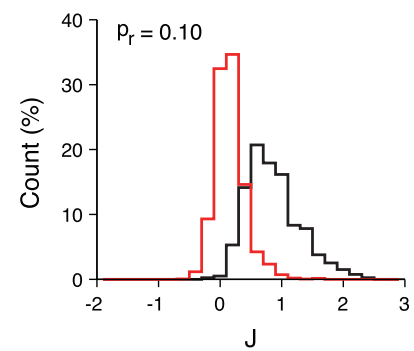

Original CERM $(\mathrm{N}=2)$
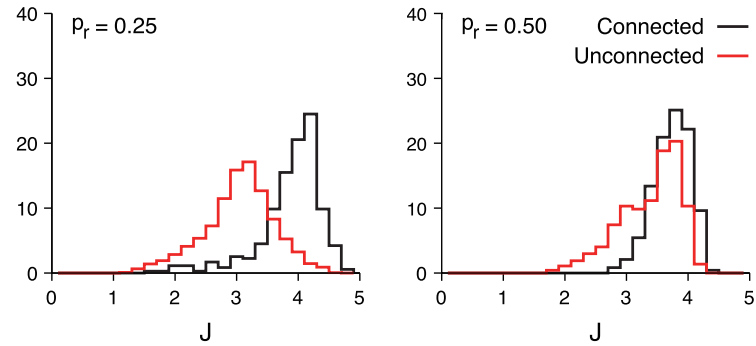

Extended CERM $(\mathrm{N}=49)$

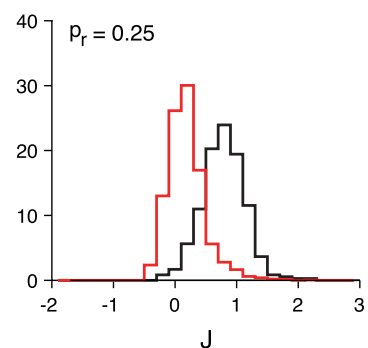

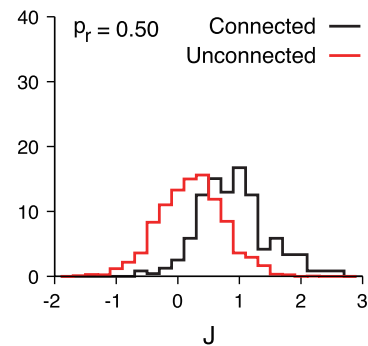

Fig. 4. Distribution of the elements of the connectivity matrix $J_{i j}$ estimated from the spike data generated by a large-scale network of Hodgkin-Huxley neurons. The connectivity was estimated by the original CERM $(N=2)(\mathrm{A})$ and the extended one $(N=49)(\mathrm{B})$. Three kinds of neural circuits with smallworld connectivity with the rewiring probability $p_{r}=0.10$ (left), $p_{r}=0.25$ (center), and $p_{r}=0.50$ (right) were examined.

connected neuron pairs was compared with the distribution for unconnected neuron pairs (Fig. 4). There were overlaps between the two distributions, indicating that it is impossible to perfectly identify the synaptic connectivity from $J_{i j}$ alone. The overlap increases when the randomness of the network topology increases (high $p_{r}$ ). We again calculated the optimal MCC between the elements of the true connectivity and its optimal estimate. MCCs obtained from the estimate by the original CERM and by the extended CERM were 0.64 and 0.68 for $p_{r}=0.1$ network, 0.55 and 0.60 for $p_{r}=0.25$ network, and 0.18 and $0.30 p_{r}=0.50$ network, respectively. The results suggested that the extended CERM performs was superior to the original CERM and that the improvement is significant in the random-like network $\left(p_{r}=0.50\right)$.

\section{Discussion}

We have proposed a method for estimating synaptic connectivity using spike data from multiple neurons (Fig. 1). We formulated the coupled escape rate model (CERM) to describe the multineuronal spike data, and the connectivity matrix was estimated by the maximum likelihood method. Finally, the synaptic connection was detected by thresholding the elements of the connectivity matrix. We demonstrated that this method could perfectly estimate the synaptic connectivity in an ideal condition, i.e., all the spiking activity was observable and the neuronal dynamics were described by CERM (Fig. 3). The result suggests that longer spike data provides more accurate estimation results, and 300-sec data is sufficient to reconstruct the synaptic connectivity from the spike data in this example. Furthermore, the proposed method was applied to more realistic spike data generated by a large-scale network of Hodgkin-Huxley neurons (Fig. 2). We investigated a partial observation scenario in which the activity of a fraction of neurons (approximately $1 \%$ ) was recorded. The proposed method performed better than the original CERM that estimate the synaptic connectivity between two neurons from spike trains of the corresponding neurons (Fig. 4). Our previous work [7] showed that the original CERM performed better than the cross-correlation and the transfer entropy method, and the original CERM requires shorter recording duration than the other methods, i.e., the estimation performance of the original CERM converged below $100 \mathrm{sec}$. These results suggest that 
the proposed method can identify synaptic connectivity better than the previous methods when the spike data from a large number of neurons is available for the analysis. One might ask how many neurons should we record to grasp the synaptic connectivity of a neural circuit. In a future work, it would be interesting to investigate how the ratio and the location of recorded neurons impact on the estimation performance.

\section{Acknowledgments}

We thank two anonymous reviewers for their valuable comments on our manuscript. This study was supported by JSPS KAKENHI Grant Number 25870915 to R.K and 15 H05877 to KK.

\section{References}

[1] Y. Ikegaya, G. Aaron, R. Cossart, D. Aronov, I. Lampl, D. Ferster, et al., "Synfire chains and cortical songs: temporal modules of cortical activity," Science, vol. 304, pp. 559-564, 2004.

[2] S. Fujisawa, A. Amarasingham, M.T. Harrison, and G. Buzsáki, "Behavior-dependent shortterm assembly dynamics in the medial prefrontal cortex," Nature Neurosci., vol. 11, pp. 823-833, 2008.

[3] M. Shimono and J.M. Beggs, "Functional clusters, hubs, and communities in the cortical microconnectome," Cerebral Cortex, bhu252, 2014.

[4] D.H. Perkel, G.L., Gerstein, and G.P. Moore, "Neural spike trains and stochastic point processes II. Simultaneous spike trains," Biophys. J., vol. 7, pp. 419-440, 1967.

[5] T. Schreiber, "Measuring information transfer," Phys. Rev. Lett., vol. 85, pp. 461-464, 2000.

[6] M. Garofalo, T. Nieus, P. Massobrio, and S. Martinoia, "Evaluation of the performance of information theory-based methods and cross-correlation to estimate the functional connectivity in cortical networks," PloS One, vol. 4, e6482, 2009.

[7] R. Kobayashi and K. Kitano, "Impact of network topology on inference of synaptic connectivity from multi-neuronal spike data simulated by a large-scale cortical network model," J. Comput. Neurosci., vol. 35, pp. 109-124, 2013.

[8] R. Jolivet, A. Rauch, H.R. Lüscher, and W. Gerstner, "Predicting spike timing of neocortical pyramidal neurons by simple threshold models," J. Comput. Neurosci., vol. 21, pp. 35-49, 2006.

[9] S. Shinomoto, "Fitting a stochastic spiking model to neuronal current injection data," Neural Netw., vol. 23, pp. 764-769, 2010.

[10] R. Kobayashi and S. Shinomoto, "State space method for predicting the spike times of a neuron," Phys. Rev. E, vol. 75, 011925, 2007.

[11] P. Dayan and L.F. Abbott, "Theoretical neuroscience: Computational and mathematical modeling of neural systems," MIT Press, 2001.

[12] D.W. Marquardt, "An algorithm for least-squares estimation of nonlinear parameters," SIAM J Appl Math., vol. 11, pp. 431-441, 1963.

[13] B.W. Mathews, "Comparison of the predicted and observed secondary structure of T4 phage lysozyme," Biochim. Biophys. Acta, vol. 405, pp. 442-451, 1975.

[14] K. Kitano and T. Fukai, "Variability v.s. synchronicity of neural activity local cortical network models with different wiring topologies," J. Comput. Neurosci., vol. 23, pp. 237-250, 2007.

[15] A. Destexhe and D. Paré, "Impact of network activity on the integrative properties of neocortical pyramidal neurons in vivo," J. Neurophysiol., vol. 81, pp. 1531-1547, 1999.

[16] A. Erisir, D. Lau, B. Rudy, and C.S. Leonard, "Function of specific $\mathrm{K}^{+}$channels in sustained high-frequency firing of fast-spiking neocortical interneurons," J. Neurophysiol., vol. 82, pp. 2476-2489, 1999.

[17] G. Buzsáki and K. Mizuseki, "The log-dynamic brain: how skewed distributions affect network operations," Nat. Rev. Neurosci., vol. 15, pp. 264-278, 2014.

[18] J.N. Teramae, Y. Tsubo, and T. Fukai, "Optimal spike-based communication in excitable networks with strong-sparse and weak-dense links," Sci. Rep., vol. 2, 485.10.1038/srep00485, 2012. 